\title{
Resettlement Experiences of Afghan Hazara Female Adolescents: A Case Study from Melbourne, Australia
}

\author{
Nida Iqbal, ${ }^{1}$ Andrew Joyce, ${ }^{1,2}$ Alana Russo, ${ }^{1}$ and Jaya Earnest ${ }^{3}$ \\ ${ }^{1}$ Cardinia Casey Community Health Service, Southern Health, Locked Bag 2500, Cranbourne, VIC 3977, Australia \\ ${ }^{2}$ Department of Occupational Therapy, Monash University, Peninsula Campus, P.O. Box 527, Frankston, VIC 3199, Australia \\ ${ }^{3}$ Centre for International Health, Curtin University, G.P.O. Box U1987, Perth, WA 6845, Australia
}

Correspondence should be addressed to Andrew Joyce, andrew.joyce@med.monash.edu.au

Received 18 July 2011; Revised 8 November 2011; Accepted 24 November 2011

Academic Editor: Cherylynn Bassani

Copyright ( $) 2012$ Nida Iqbal et al. This is an open access article distributed under the Creative Commons Attribution License, which permits unrestricted use, distribution, and reproduction in any medium, provided the original work is properly cited.

\begin{abstract}
Young people from refugee backgrounds face a number of challenges in adjusting to life in a new country. Recently, there have been more studies documenting some of these challenges and experiences, and offering recommendations for the health and education sector to appropriately respond to their needs. This study sought to investigate some of the experiences and challenges faced by female Afghan Hazara refugee adolescents as a precursor to program development occurring within a community health service in the outer southeastern suburbs of Melbourne, Australia. This paper reports on a cross-sectional participatory qualitative research study undertaken with young Afghan female adolescents aged 14-17 years of Hazara ethnicity. The results document some of the key contested gender and cultural challenges facing these young women, their aspirations for their lives in Australia, and how this research has informed community health practice.
\end{abstract}

\section{Introduction}

Individuals from Culturally and Linguistically Diverse (CALD) backgrounds confront a variety of unique problems when adjusting and resettling to life in a new country. For individuals from refugee backgrounds, difficulties associated with language and cultural differences are frequently compounded by prior experiences of discrimination, stigma, human right violation, and trauma which potentially exacerbate mental health problems. Research conducted with refugee women in South Australia indicated that transitional experiences upon arrival in Australia often did not meet their expectations. Whilst coming to Australia presented the chance of survival, a variety of unexpected challenges contributed towards a sense of hopelessness, often resulting in low self-esteem and depression [1]. Young people from refugee backgrounds in particular face a number of resettlement challenges, and while burgeoning research into how this process unfolds for refugee health is emerging globally [2] there is little information about this transition for Afghan female youth. This study addresses this gap focusing on refugee female adolescents from the Hazara ethnic background.

\section{Literature Review}

A qualitative study of the social and emotional wellbeing of 123 young people from CALD backgrounds living in Queensland, South Australia and Western Australia included 76 people from refugee backgrounds [3]. The interview data of the participants from refugee backgrounds was analysed separately to the other participants, allowing specific exploration of the unique emotional and social issues arising due to the compounding experience of arriving as a refugee. The findings indicated that the traumatic past of young people was something that continued to influence their lives within Australia. Experiences of racism within Australia were correlated with depression and social withdrawal, as they triggered memories of violence from their past. Social connection with members of their own cultural community has been identified as an effective form of comfort for people from CALD and refugee backgrounds. 
Whilst young people from CALD and refugee backgrounds have indicated enjoying and appreciating the freedoms within Australia, these have also been identified as a source of conflict, contrasting with family responsibilities and roles from their cultural backgrounds. Conflicting values, traditions, customs, and expectations between young people and their families have been identified as a common cause of family disruption [4]. Relationship conflict between generations is a source of stress for many young people [3]. This has been further demonstrated through a study conducted by Kids Helpline which indicated that young people from Non-English Speaking Backgrounds (NESB) called the help line to discuss family and relationship problems at rates exceeding those of the broader English speaking population. The majority of calls from NESB young people were in regards to family relationship problems arising from intergenerational conflict and peer relationship difficulties related to frequent and severe bullying, and language and cultural difficulties [4].

Young people from CALD and refugee backgrounds also face a variety of educational challenges $[5,6]$. The demanding supportive roles which these young people take within their homes and the disrupted or lack of formal education within their respective countries make schooling challenging and very difficult [3]. This was reiterated in findings with high school students from Southern Sudanese backgrounds who encountered schooling difficulties due to the loaded responsibility of looking after their families compounded by low levels of education within their respective country [7]. The difficulties highlighted were found to decrease the likelihood of successful transition to tertiary study, despite aspirations for further education. Young people from NESBs also experience increased discomfort within the school environment, as a result of being subjected to direct and indirect discrimination and stigmatisation due to colour, accent, dress, and an initial lack of understanding of the Australian education system [4].

A quantitative study of the predictors of education success among 91 refugee youth in Canada found that just over half (53\%) were doing well in high school [8]. Success in this study was defined as having successfully completed high school or being at high school in the correct grade in relation to their age and self reporting no current difficulties. Grade placement on arrival, increased length of time in Canada, ethnicity, urban residence, and parents' health were the significant factors in a regression model. Recent research tracking the health and wellbeing of refugee youth over a three-year period concluded that their perceived social status of their community, discrimination and bullying were key factors influencing wellbeing and that policies and programs need to address the broader social environment to ensure successful outcomes for these young people [2].

Whilst youth from refugee backgrounds share some similar experiences, it is imperative that they are not considered a homogenous group. Each refugee community has distinct and unique characteristics. And the levels of wellbeing have been found to differ among ethnicities for refugee young people [2]. Melbourne's outer southeastern suburbs have recently experienced an increase of population groups from
CALD backgrounds, many arriving on humanitarian visas. The largest refugee group within the City of Casey where this study took place is the Afghan population with 1703 people according to 2006 census, although this figure would have increased by the 2011 census the results of which are not yet released [9]. The participants of this study were primarily comprised of people from Hazara ethnicity.

Afghanistan has an intricate history of turmoil over four decades which has included war and discrimination on the bases of race, religion, and gender resulting in the largest population movement in modern times. Many of the current Afghan youth living in Australia have been born during the Taliban years which were characterised by extreme Islamic practices which had devastating health and education consequences especially for Afghan women [10]. Under the Taliban rule women were forbidden to work, attend school, or leave the house unaccompanied by a male family member. Women were required to wear a burqa which covered them from head to toe with only a net over their eyes. Women who did not comply with these regulations were severely punished, often in the form of public beatings [10].

These violations of women's rights were justified in the name of culture and religion. It is then of particular interest to consider the experiences of Afghan youth resettled in Australia, as they were born in a volatile and violent country at a time coinciding with the early stages of the Taliban regime. The impact of this harsh and complex environment has direct effects on the developing child and indirect effects through impacts on their mothers and other significant carers. Thus it is important to consider the challenges posed by the traumatic experiences faced by Afghan youth especially young women combined with contrasting cultural and gender values when resettling into Australia.

Community support has been identified as benefiting the health and wellbeing of youth from CALD and refugee backgrounds [3]. Furthermore, Brough et al. concluded that conceptualising the experiences, health, and wellbeing of this population group does not fit well within the boundaries of the biomedical model of health and illness [3]. Rather, the way which the participants articulated their lived experiences exemplified "the importance of promoting supportive social environments within public health approaches to refugee health. Community development strategies which connect young people to communities and communities to young people are of critical importance" (page 206).

There are a range of barriers which hinder the development and implementation of health promotion programs for people from CALD communities. Research consistently reiterates the importance of culturally appropriate programs and strategies which acknowledge the diversity within CALD communities also. Services and messages must extend beyond translation into the language of the communities which they attempt to engage, to align with the values inherent within the cultures of these population groups [11]. It is also important to consider some of the structural and societal barriers that may be hindering the wellbeing of these young people and how interventions can address these factors [2]. While there are general recommendations for working with these communities, at this stage, there is a lack 
of coordination, sustained support, and evidence base for programs aimed at promoting the health of refugee young people [12].

There have been recent calls for the research and development of more coordinated and comprehensive programs for refugee youth $[12,13]$. There is an unequivocal need for research that ascertains the psychosocial needs of refugee youth. This reaffirms the need to engage in consultation with various population groups and explore the unique experiences and situations of these newly arrived peoples in order to respond with appropriate, accessible, and wellresourced services [4]. While there has been some innovative and informative research with Afghan male youth as part of the Good Starts Arts project [14], there seems to be less information available about Afghan female youth.

\section{Project Aims}

There is limited research exploring the health and social issues and needs of Afghan adolescents from refugee backgrounds and little information about the experiences of female Hazara adolescents. Consequently, educators and social health workers have limited knowledge about the unique experiences of this group, making it difficult to respond to the health and social wellbeing needs of this growing population. Therefore, this cross-sectional research explored the settlement issues of female Hazara adolescents, examined the challenges they face and their aspirations for the future. Recommendations drawn from the analysis will contribute towards the knowledge base of youth from refugee backgrounds.

\section{Methods}

4.1. Research Design and Methods. This study was conducted to inform the development of community health and social programs to better support refugee youth, in particular Afghan females from Hazara ethnicity within mainstream secondary school. Recruitment and data collection was undertaken by the first named author as part of her employment with Cardinia Casey Community Health Service, Melbourne, Australia. It was considered pivotal to the project that someone familiar to these young people undertake the interviews and that through this rapport ongoing involvement in programs could be achieved. This fostering of participatory relationships was one of the key principles underpinning the methodology [15]. The study utilised qualitative gendered research methods drawing on life history approaches that involved a sharing of stories and drawing interpretations [16].

Using some of the recommended strategies from Baur et al. [17] in conducting research with marginalised groups, informal conversations were conducted with the prospective participants before the interviews took place. Participants were visited at least one week before the in-depth interviews and informed of the semistructured questions that were part of the interview schedule. They provided feedback about the types of questions they felt comfortable to answer and provided some information about their current experiences in Australia. Through this informal consultation the participants asked the first author about her background and aspirations in Australia, as the first author has a refugee background and is of Hazara descent, came to Australia in her late teenage years over a decade ago, and is now a community support worker. This building of rapport and trust was considered pivotal in securing the confidence of the participants for this study and involvement in future programs which have evolved in the form of a peer support group.

This paper draws on the voices and the overall experiences of Hazara females from refugee backgrounds based on their histories, resettlement experiences, family situation, social and cultural factors, and study experiences within their countries of origin and within Australia from the audio recorded interviews.

4.2. Participants. Eight in-depth, semistructured interviews were conducted with Afghan Hazara female participants between the ages of 14 and 17 years. The participants were studying at three different government secondary schools in Melbourne's southeastern suburbs. Following the in-depth interviews, a focus group discussion was held with eight female participants, two of whom had previously taken part in an interview. Participants were pooled from personal contacts initially, with snowballing and purposive sampling occurring thereafter. The inclusion criteria was arriving in Australia within the last five years and although it was not the original intent to only interview those of Hazara ethnicity this eventuated due to the other Afghan ethnic groups in the area having been settled for a longer period of time.

4.3. In-Depth and Focus Group Questions. The semistructured in-depth interviews and focus group discussions were guided around specific categories such as:

(i) demographics (including ethnic background and socioeconomic factors),

(ii) experiences in Afghanistan and migration to Australia,

(iii) school environment (learning, teaching, and social) and experiences at school and at home,

(iv) negotiating identities between Australian and Afghan culture,

(v) peer relationships,

(vi) family relationships,

(vii) future aspirations in Australia.

These categories were identified in order to assess aspects of the students' resettlement into Australia, in particular the unique issues arising from being a female teenager and adolescent from a Hazara refugee background living within Australian society. The dialogue was planned to cover issues broadly and not just focus on the past experiences of trauma and its effects on the present, rather this was considered just one of many aspects to the lives of these young people [14]. 
The interviews and focus groups were conducted in Dari and as the first named author is an accredited interpreter, they were transcribed in English. Even though many of the participants were fluent in English the use of Dari rather than English was considered an important rapport building strategy. All the interviews and focus group took place between July and September 2009.

4.4. Ethical Approval. Ethics approval for the project was obtained from the Human Research Ethics Office at Curtin University and the Standing Committee on Ethics in Research Involving Humans through Monash University.

4.5. Thematic Content Analysis. After the interviews were completed and transcribed a thematic content analysis was undertaken to collate and condense the information gathered into distinct, and succinct themes $[18,19]$. The aim of the analysis was to produce a thematic content analysis that was succinct and reliable and could display the key themes in an easily communicated manner to a nonresearch literate audience [20]. This was considered an important tool in communicating the results to participants, as the summarised themes and recommendations were provided to participants for their feedback, and then provided to staff and managers of the health service from which appropriate strategies have been planned and delivered. Based on this feedback the themes were further refined by the research team.

\section{Results}

5.1. Perception of School. The participants identified several educational and social difficulties such as language and bullying within school which will be later discussed. Despite these difficulties, participants consistently stated that they valued education and wanted to go to school. They felt that the school environment was safe, they enjoyed school and believed that the teachers acted in the best interests of the students, offering academic and social support. Students expressed gratitude for the peaceful school environment,

\begin{abstract}
Students are not fearful of teachers. In Afghanistan, teachers are seen as the highest form of authority and everyone is scared of them. In Australia they are friendly and not scary at all. [Participant 3, 14 years].

In Australia, schools think about children and that they should be at peace and have a good time...[I] look forward to going to school every day. [Participant 1,14 years].
\end{abstract}

5.2. Educational Difficulties. Despite their appreciation for the school environment, participants stated that school was one of the hardest things about settling in Australia. Limited English and lack of prior formal education within their respective countries were identified as barriers to education within Australia. The participants expressed frustration in not understanding and not being understood. However, some frustration decreased upon familiarity with the English language, reinforcing previous assertions that six to 12 months of ESL services for newly arrived migrants are inadequate for young people with disrupted schooling backgrounds, resulting in immediate frustrations for teachers and students [21],

\section{It was hard until I learnt English because I had not received any education when we were in Iran...It was the first time I went to school when we came to Australia. [Participant 5, 15 years].}

These experiences of the participants are likely to be similar to those of many recent humanitarian arrivals into Australia from non-English speaking backgrounds; however, there are peculiarities specific to this population group, reflecting the political culture of Afghanistan and the denial of educational opportunities to women.

5.3. Social Difficulties. The participants consistently expressed social difficulties at school. This included explicit forms of bullying such as name calling and teasing, in which people referred to them as "unsocial" or "terrorists." The issue of clothing emerged within each interview, in which the females felt separated from their peers as their attire immediately identified them as Afghan, and therefore, as different. Bullying often related to the wearing of the traditional headscarf. Several comments indicated that other students had pulled off their head scarfs, and that they had been interrogated as to why they bother wearing the scarf when some hair could be seen at the front.

More subtle forms of social difficultly included having trouble mixing with peers, feelings of dislike by other members of their class, and having no one to partner with for paired or group class activities. The young women further expressed feeling inferior to the broader, predominately Western population, believing that others look down on them and avoid contact with them. The traditional Afghan names were also identified as a topic for school bullying,

We have difficulty in mixing with people because of the way we're dressed...I always feel that people look down at me. [Participant 6, 16 years].

Because of the way we dress...we have been called a terrorist or unsocial people. People refuse to talk to us or have a relationship with us. [Participant 8,15 years].

When I started school with my older sister, we didn't know enough English. Two boys used to take our scarfs off. So she left school. After a few years she started going to TAFE. [Participant 4, 16 years].

5.4. Culture and School. The participants identified several cultural differences which contributed towards uncomfortable school experiences. These included learning about the body and sex education in front of male peers. Whilst participants indicated an understanding of the importance 
of this education, and a general appreciation for this, they found having these discussions around boys embarrassing. Other differences included playing sport and going on school camps and school excursions. According to the participants these are considered taboo within Afghanistan and inconsistent with the culture of this population group, despite being considered normal within Australian society,

Learning about my body in front of boys was very embarrassing. I didn't like going to school on those days. [Participant 2, 17 years].

5.5. Family Support and School. Participants identified several difficulties associated with school experiences within Australia and the culture of their family life. There was a variety of responses relating to how much parents supported adolescent female education. Whilst some females expressed that their parents were very supportive of their education, wanting them to continue onto successful careers, others stated that education was not considered a priority for Afghan females, but rather that their duties were within the household. While the participants were all of Hazara ethnicity, in dialogue they made a number of general comments in relation to Afghan culture broadly when discussing family and gender roles.

This was a frustration for some participants, as they consistently indicated aspirations to continue studying and pursue careers. Although the participants were all enrolled within school, they stated that many girls within their cultural community were not allowed to have an education, having the cultural practices of their respective country imposed upon them. Within Australia, education is considered to be a predictor for future employment and economic status. Some participants reflected that they felt the cultural perspectives of Afghanistan were impeding their social, economic, and educational opportunities, potentially disposing them to a variety of health and social problems,

The main problem for Afghan girls is that they want to have education and go places but in Afghan culture, they have to stay home. [Participant 7, 14 years].

Fifty percent of families don't allow their daughters to go to university and have a career. [Participant 8,15 years].

Some girls want to receive education, but parents think that you should have limited education and then learn household skills and go and marry a boy and have kids. Some parents don't care about their daughter's lives, but the girl might want to have an education and a career. [Focus group comment].

The issue of whether males were allowed more freedom within school and given increased educational opportunities was a reoccurring theme within each of the interviews and one of main topics of conversation in the focus group. The participants expressed frustration with living in Australia under what they perceived as Afghanistan's cultural structure of gender roles. The overarching gender issue was the higher status and value of males within their families and cultural communities. The participants identified males as having more freedom and enjoyment while females were required to stay home performing domestic duties. Participants stated that families listen to boys more, support their education more, and allow them more freedom than girls,

Parents and families cannot stop boys from doing all these things because they are boys and they have more value and status in a family than a girl. [Focus group comment].

Boys can have lots of fun, but girls have to stay at home and do housework. . it should be the same for boys and girls. [Focus group comment].

Some families want their girls to have a little bit of freedom but they cannot do it because of other people. They will have to listen to other people. [Focus group comment].

Young women within Afghanistan have experienced significant inequalities and repression for many years and according to these participants this cultural norm has, to a certain extent, been transferred into their lives within Australia. Several participants stated that their parents were not supportive of some school activities, in particular physical education, sex education, and school camps. Participants stated that parents were not supportive of their daughters participating in sporting activities, as such is considered incongruent with Afghan culture, especially when this may involve close proximity or contact with males. Attendance to school camps was forbidden for similar reasons,

...[I] cannot tell my parents that I love PE because...mum thinks that PE is not for girls. . she says that I shouldn't do sports because boys and girls play together... I have never been allowed to go on camps. [Participant 1, 14 years].

I never spoke about this [sex education] at home. If I did, my mum would cry and my dad would go to school and fight with teachers and tell me to stay home. [Participant 2, 17 years].

Finally, participants stated that it was difficult to discuss school issues within their homes. Problems such as bullying, social, and academic difficulty were kept to themselves as these participants felt that their parents would not be familiar with these issues and unable to be of assistance. One participant expressed that Afghan culture does not discuss any problems within the home, often leaving individuals to carry such burdens alone,

If something happens outside the home, because of our culture and being shameful, we cannot tell anyone at home. [Participant 5, 15 years]. 
5.6. Family Responsibility. Several family responsibilities were identified as affecting the participants. Issues of family violence, both physical and verbal, arose in several discussions and were identified as adversely impacting the behaviour of the children within the home. The participants also indicated high levels of responsibility relating to household duties, managing family finances, assisting parents and siblings, and interpreting for non-English speaking parents. The pressure of this was identified as adversely impacting their education and social wellbeing. This is consistent with previous research on refugee youth within Australia. Furthermore, existing within the community, in particular, was the notion of shame and honour within Afghan culture. Shame and honour were identified as placing responsibility and pressure on young adolescent females in relation to gender roles,

Lots of family responsibility on me, I have to look after all the bills for home and also do interpreting for them at times...I know that sometimes it is not appropriate for me to act as an interpreter. Some organisations don't like it as well. It is not professional and ethical and I might not know the appropriate language and I'm a young adult. [Participant 2, 17 years].

It is hard for both girls and boys, but because of cultural restrictions and belonging to a honour and shame based culture, girls are scared of following the path of becoming an Australian, but boys do it more freely and they don't have a fear. [Focus group comment].

5.7. Marriage. Participants expressed some coercion to marry at a young age, with suggestions of pressure to marry within their cultural community or to agree to an arranged marriage. This appeared to be closely related to cultural gendered roles, in which girls were expected to marry the same way as their parents did in Afghanistan. This was identified to be a cause of conflict between young females and their parents, and a contributor to poor mental health among many female Afghan youth.

One participant stated that girls within the Afghan community may "attempt suicide if they are forced into an arranged marriage." Whilst the term suicide is stated with caution, this is indicative of how seriously these girls perceive the issues of forced marriage, consistently indicating the desire to choose their partner and not conform to the Afghan tradition of arranged marriage,

Some parents don't even want to ask their daughters if they want to marry a man. The parents will choose a man for their daughters. We believe that parents should ask their daughters if they want to marry a man. [Focus group comment].

5.8. Past Trauma. Several participants indicated that the traumatic experiences of their pasts including premigration experience and their refugee journey still pervaded their thoughts and affected their emotions. This is consistent with previous research which indicates that the history of being a refugee continues to affect people's lives within their new country. These findings reiterate the need to consistently respond to the unique mental health needs of refugees within Australia. Despite being affected by the trauma of their past, the female youth expressed a general appreciation for the cohesion, the peace, the security, and freedom of choice that exist in Australian society,

There are times that I can't stop thinking about what we all went through, losing our family members and losing my brother. [Participant 3, 14 years].

At times when everything is quiet, I sit and think about the peace we have here and hopefully [the torture and the fear] will never happen again. [Focus group comment].

5.9. Social Wellbeing. The participants indicated social difficulty in which they felt excluded and found it hard to make friends outside of the Afghan community. This was attributed to cultural differences in which the broader Australian population was considered to be uninformed about Afghan culture, creating a barrier to friendship. Participants consistently stated that much of the social exclusion came from looking different due to the clothing (such as wearing the head scarf or hijab) that they are culturally required to wear,

It is harder in the society to become friends with people outside our community because they don't have enough knowledge about our culture and they don't know about some of our customs. [Focus group comment].

Sometimes.. the bus drivers treat us differently or if we are in a park then other people try to avoid us or distance them from us so that they are not close to us. [Participant 5, 15 years].

5.10. Negotiating between Cultures. The participants consistently indicated emotional struggle in negotiating between Afghan and Australian culture, discussing the difficulty in being caught between the two worlds and feeling partial connectedness and responsibility to both. Whilst this was explicitly stated within the majority of interviews, it was also underscoring many of the aforementioned findings,

I want to keep bits and parts of my culture in Australia and live parts of both worlds. [Participant 1,14 years].

It has been hard in Australia...I have family pressure and sometimes it gets very difficult for me to live in two worlds. [Participant 2, 17 years].

5.11. Future Aspirations in Australia. Although as stated the participants were pessimistic about some of the opportunities available to other Afghan female adolescents, many of the 
participants expressed a strong desire to achieve education and career success and overcome any obstacles present. Some of the participants appreciated their own parents' support towards their education and career aspirations and thus the participants' tendencies to generalise about the imposition of Afghan culture on gender roles was not necessarily reflected in their own families,

Some families don't know what freedom is. They think freedom is clubbing or having a boyfriend. But to us freedom is to receive education and go and work. [Focus group comment].

For me, there will be many challenges and hurdles and hopefully I will be able to face them and have strength. [Participant 3, 14 years].

My parents want me to have a good future and have a good career in my life. They don't want me to marry young, have kids and live my life like other Afghan women...My parents provide me lots of help with my education and future. [Participant 2, 17 years].

I'm lucky that they [parents] want me to have education and my freedom is important to me and they want me to have a good career. [Participant 1,14 years].

\section{Discussion: Program and Policy Implications}

This study is limited in its selection of participants from a specific ethnic group within a defined local government area within Melbourne, Australia. While considered a vital strategy to accessing a population group that has not been well researched, having an interviewer with a shared experience as the participants could also be contented as producing a strong element of bias into the conduct of the interviews and analysis. The participants also expressed a number of broad comments in relation to cultural practices. While it is important to capture how the Hazara female youth understood their social environment, the degree to which this is reflective broadly of the experiences of Afghan female youth from other ethnic backgrounds (Pashtun, Uzbek, Balochi, and Turkmen) and refugee female youth from other countries cannot be assumed. However, many of the findings were consistent with the experiences of refugee youth as identified within previous research. Similarities included educational difficulty due to increased responsibility within the home, lack of prior education in respective country, and language barriers [3]. Similar to other research these young people had experienced discomfort with the school environment as a result of being subjected to direct and indirect discrimination [4].

In comparison to young Afghan males there were some similarities in findings such as language challenges, negotiating between two cultures, trying to fit within their new society, and appreciation coupled with suggestions of apprehension of the freedoms found in Australia [14]. There were also a number of differences between the genders. Whereas males have an ability to dress in a "safe way" that would not gain unwanted attention [14] this was not something that many of the female participants could achieve. Many of the participants commented on the different roles expected of them at home and school relative to the experiences of their brothers and other young males.

The findings of this research led to the development of a peer support group in 2010 so that these adolescents could discuss and support each other with some of the cultural and gender related challenges outlined in this research. Previous research has found that East Timorese people felt that social workers empowered their children which often conflicts with traditional Timorese societal and cultural mores [22] and this has also been an experience of refugees from some African communities [23].

Given these findings, it was considered important that a female Hazara community member facilitate the group so that cultural appropriateness, safety, and interpretation were embedded within the group program. An adolescent psychologist from a Caucasian background cofacilitated the group and the group was drawn from within a secondary school so that appropriate partnerships, collaboration, and coordination were implemented, so that foundations for ensuring appropriate and successful programs and services are established $[12,24]$. Given that some of the participants commented that they were unable to attend sex education classes, issues related to relationships, sex, and STIs were covered in the group among other topics. This study found that similar to previous research there is a lack of knowledge and information access around sexual health for people from refugee backgrounds, and the importance of providing this information in a flexible, sensitive, and culturally appropriate manner is vital $[25,26]$.

While issues of gender roles and family responsibility restricting educational opportunities are common findings across a number of studies $[3,5,6]$, there seemed to be more pessimism about opportunities for educational and career attainment among these Hazara participants compared to research conducted with South Sudanese young people [7]. Thus as mentioned, in this study, a Hazara female tertiary educated community member cofacilitated the group, and Hazara women currently enrolled in tertiary education or working were invited to attend the group.

This research has also outlined strategies that the schools and community centres could implement to promote a more inclusive environment for these Hazara students and potentially CALD students from other backgrounds. Intercultural education is perceived as being important for all children and is frequently endorsed as a facet of good education [27]. Schools have been identified as an effective forum to promote and facilitate interculturalism, assisting children to develop positive intergroup relations, incorporating principles of respect, understanding, and acceptance [27-29]. Evaluations and meta-analyses of interventions consistently indicate positive outcomes among students [30, 31].

Furthermore, benefits of intercultural, interfaith, valuesbased education extend beyond reductions in racial bias, to include a range of improvements including increased 
responsible behaviours, academic diligence, and enhanced student-and-teacher relationship and wellbeing [32]. Direct, explicit intercultural education has previously been the dominant form of interventions, however, the implicit interactional approach has been gaining credibility [27]. The interactional approach involves intentionally bringing diverse groups together to encourage positive interactions within the safe, protective school environment [33]. This can involve sporting activities, social activities, and other intercampus events [30]. Research has indicated that intercultural interaction is positively related to the promotion of racial understanding and reduction of racial bias, with additional benefits including improvement in school satisfaction, social self-confidence, and critical thinking skills [30]. However, it must be noted that positive intergroup contact relies significantly on the provision of appropriate conditions. When supportive conditions are not in place, the interaction has the potential to have an adverse effect in which racial bias may be heightened [30].

Based on the results of this study in the peer support program students were assisted in developing their selfconfidence to talk at a school assembly about features of their culture such as wearing of scarfs and the challenges in mixing with boys during certain school activities. The students were provided with lots of practice about the type of messages that would assist in discouraging discrimination and after providing a presentation in assembly according to their feedback this increased the respect they were shown by the school community and greatly reduced the level of bullying and harassment they received. This approach is consistent with recommendations for strategies that ensure inclusive environments for refugee young people [2], and further research is planned to evaluate systematically whether similar types of interventions can reduce the frequency of bullying behaviour.

\section{Conclusion}

The findings of this study highlight the importance of maintaining and building connections with members of their own community and with the broader community. Many of the cultural impacts identified in this study are related to being female, as this potentially influences the clothing that many of them wear, the activities they can participate in, and the amount of educational support they receive from their family. This can compound the more common experiences of young people from refugee backgrounds in negotiating between two cultures, causing intergenerational disputes and creating a feeling that they do not completely belong to either community. Varying forms of extreme trauma and mental health issues as a result of human rights violations are a commonality, identified within previous research and reiterated within these findings [3]. Further research is required to investigate participants' claims that adolescent girls are not being allowed to attend higher secondary school or to be tertiary educated and are kept at home by their parents.
This study has highlighted some of the issues faced by young Hazara women during resettlement and how this can inform program development. It was clear from this research that Hazara young women needed more positive and empowered female role models not only from their community but also from the wider migrant community. The participants of this study were highly motivated to successfully complete secondary education and undertake future study in pursuit of rewarding careers. Further research is required on the efficacy of programs and policies for refugee young people to ensure successful access to educational and social opportunities so that their ambitions can be realised.

\section{Acknowledgments}

The authors would like to thank the participants for their contribution to this study and participation in the peer support group. They acknowledge Southern Health for providing a workplace environment that fosters research and supports practitioners to undertake participatory action research that promotes programme development.

\section{References}

[1] D. Costa and J. Williams, "New arrival refugee women, health and wellbeing project," Synergy, p. 9, 16-18, 2002.

[2] I. Correa-Velez, S. M. Gifford, and A. G. Barnett, "Longing to belong: social inclusion and wellbeing among youth with refugee backgrounds in the first three years in Melbourne, Australia," Social Science and Medicine, vol. 71, no. 8, pp. 13991408, 2010.

[3] M. Brough, D. Gorman, E. Ramirez, and P. Westoby, "Young refugees talk about well-being: a qualitative analysis of refugee youth mental health from three states," Australian Journal of Social Issues, vol. 38, no. 2, pp. 193-208, 2003.

[4] D. Incorvaia, "Protective and risk factors in the mental health of migrating young people from non-english speaking backgrounds," Connect, Mental Health Matters in WA, vol. 6, no. 3, pp. 24-25, 2001.

[5] J. Earnest, A. Joyce, G. De Mori, and G. Silvagni, "Are universities responding to the needs of students from refugee backgrounds?" Australian Journal of Education, vol. 54, no. 2, pp. 155-174, 2010.

[6] A. Joyce, J. Earnest, G. de Mori, and G. Silvagni, "The experiences of students from refugee backgrounds at universities in Australia: reflections on the social, emotional and practical challenges," Journal of Refugee Studies, vol. 23, no. 1, pp. 82-97, 2010 .

[7] E. Cassity and G. Gow, "Making up for lost time: the experiences of Southern Sudanese young refugees in high schools," Youth Studies Australia, vol. 24, no. 3, pp. 51-55, 2005.

[8] L. Wilkinson, "Factors influencing the academic success of refugee youth in Canada," Journal of Youth Studies, vol. 5, no. 2, pp. 173-193, 2002.

[9] City of Casey, "Bithplace Countries City of Casey Community Profile," 2010, http://profile.id.com.au/Default.aspx?id= $109 \&$ pg=103\&gid=10\&type $=$ enum.

[10] Z. Rasekh, H. M. Bauer, M. M. Manos, and V. Iacopino, "Women's health and human rights in Afghanistan," Journal 
of the American Medical Association, vol. 280, no. 5, pp. 449455, 1998.

[11] M. Moore, D. Lane, and A. Connolly, One NESB Size Does Not Fit All! What Makes a Health Promotion Campaign "Culturally Appropriate"? Australian and Transcultural Mental Health Network, 2002.

[12] K. Zwi, S. Raman, D. Burgner et al., "Towards better health for refugee children and young people in Australia and New Zealand: The Royal Australasian College of Physicians perspective," Journal of Paediatrics and Child Health, vol. 43, no. 7-8, pp. 522-526, 2007.

[13] E. Thorell, Attending to the Needs of Refugee Youth: The Development of a National Comprehensive Youth Service, Centre for Refugee Research, University of New South Wales, Sydney, Australia, 2007.

[14] A. Rodríguez-Jiménez and S. M. Gifford, ''Finding voice': Learnings and insights from a participatory media project with recently arrived Afghan young men with refugee backgrounds," Youth Studies Australia, vol. 29, no. 2, pp. 33-41, 2010.

[15] S. M. Gifford, C. Bakopanos, I. Kaplan, and I. Correa-VVelez, "Meaning or measurement? Researching the social contexts of health and settlement among newly-arrived refugee youth in Melbourne, Australia," Journal of Refugee Studies, vol. 20, no. 3, pp. 414-440, 2007.

[16] F. Baum, The New Public Health, OUP, Melbourne, Australia, 3rd edition, 2008.

[17] V. E. Baur, T. A. Abma, and G. A. M. Widdershoven, "Participation of marginalized groups in evaluation: mission impossible?” Evaluation and Program Planning, vol. 33, no. 3, pp. 238-245, 2010.

[18] K. Neuendorf, The Content Analysis Guidebook, Sage, Kern County, Calif, USA, 2002, Edited by K. Neuendorf.

[19] R. Boyatzis, Transforming Qualitative Information, Sage, Kern County, Calif, USA, 1998.

[20] H. Reis and C. Judd, Handbook of Research; Methods in Social and Personality Psychology, Cambridge University Press, Cambridge, UK, 2000.

[21] L. Olliff and J. Couch, "Pathways and Pitfalls: the journey of refugee young people in and around the education system in Greater Dandenong, Victoria," Youth Studies Australia, vol. 24, no. 3, pp. 42-46, 2005.

[22] Piscitelli, "In their own words," Synergy, p. 7-8, 32-34, 2000.

[23] J. Q. Nyagua and A. J. Harris, "West African refugee health in rural Australia: complex cultural factors that influence mental health," Rural and Remote Health, vol. 8, no. 1, p. 884, 2008.

[24] S. B. Murray and S. A. Skull, "Re-visioning refugee health: the Victorian Immgirant Health Programme," Health Services Management Research, vol. 16, no. 3, pp. 141-146, 2003.

[25] C. McMichael and S. Gifford, "It is good to know now...before it's too late," Sexuality and Culture, vol. 13, no. 4, pp. 218-236, 2009.

[26] C. McMichael and S. Gifford, "Narratives of sexual health risk and protection amongst young people from refugee backgrounds in Melbourne, Australia," Culture, Health and Sexuality, vol. 12, no. 3, pp. 263-277, 2010.

[27] Y. Leeman, "School leadership for intercultural education," Intercultural Education, vol. 14, no. 1, pp. 31-45, 2006.

[28] M. E. Engberg, "Improving intergroup relations in higher education: a critical examination of the influence of educational interventions on racial bias," Review of Educational Research, vol. 74, no. 4, pp. 473-524, 2004.

[29] L. Johnson, "The diversity imperative: building a culturally responsive school ethos," Intercultural Education, vol. 14, no. 1, pp. 17-30, 2003.
[30] N. Denson, "Do curricular and cocurricular diversity activities influence racial bias? a meta-analysis," Review of Educational Research, vol. 79, no. 2, pp. 805-838, 2009.

[31] S. Oskamp, Reducing Prejudice and Discrimination, Lawrence Erlbaum Associates, 2000.

[32] T. Lovat, K. Dally, and N. Clement, "Project to Test and Measure the Impact of Values Education on Student Effects and School Ambience," 2009, http://www.curriculum.edu.au/ verve/_resources/Project_to_Test_and_Measure_the_Impact_of_ Values_Education.pdf.

[33] P. Batelaan, "Learning to respect," Intercultural Education, vol. 12, no. 3, pp. 237-245, 2001. 


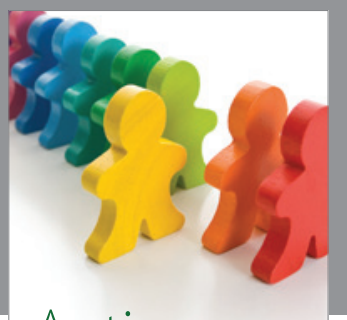

Autism

Research and Treatment
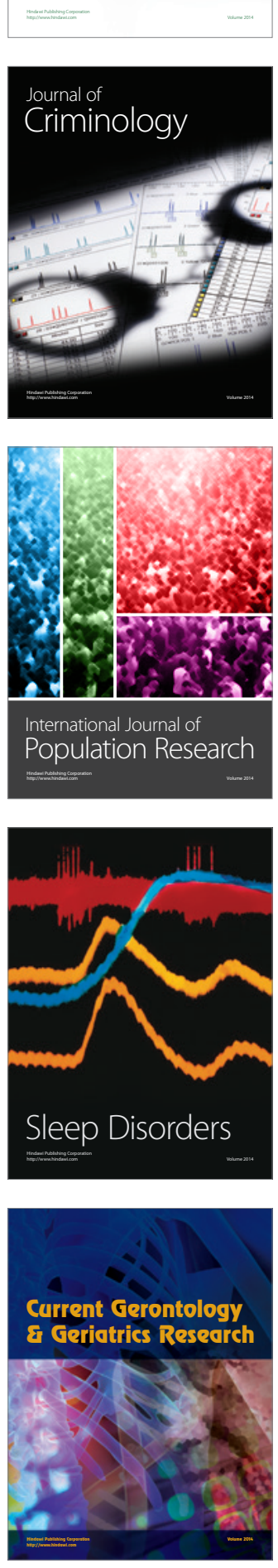
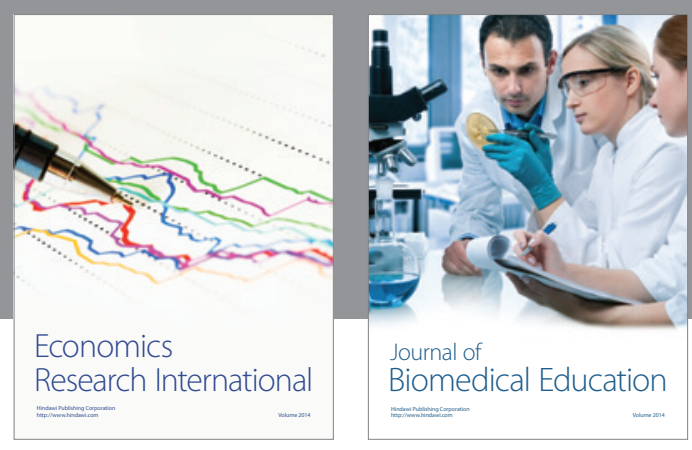

Journal of

Biomedical Education

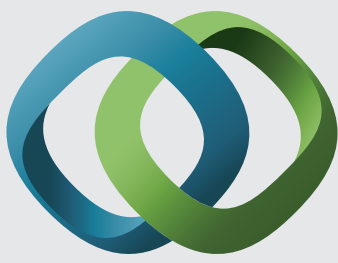

\section{Hindawi}

Submit your manuscripts at

http://www.hindawi.com
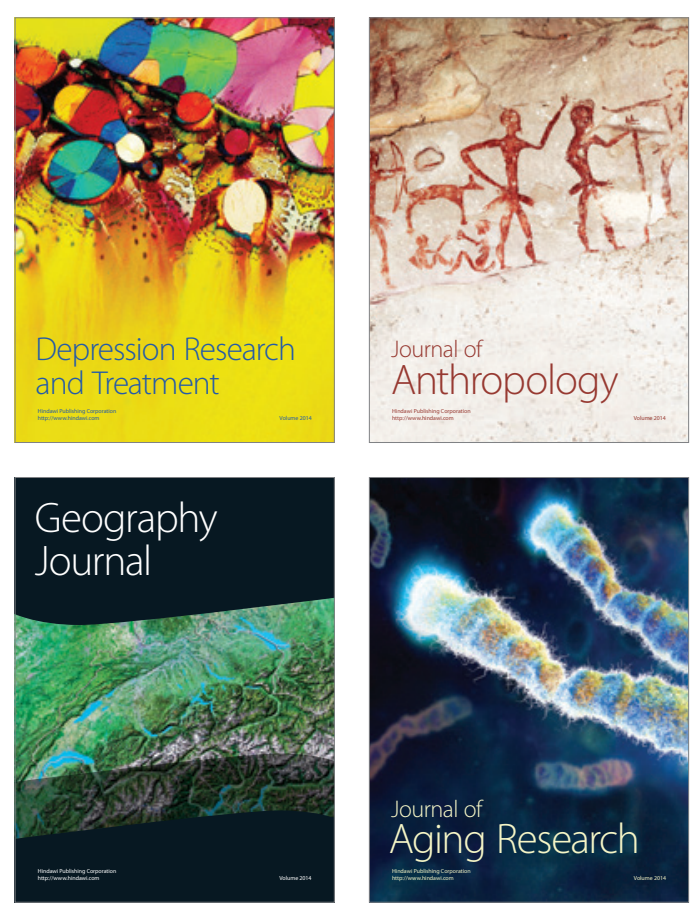

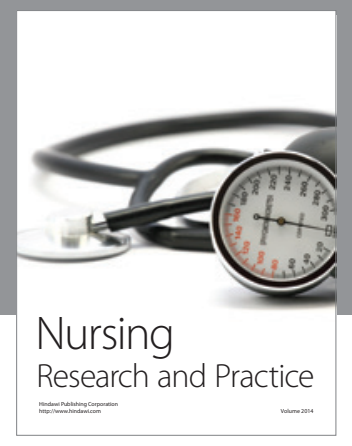

Nursing

Research and Practice

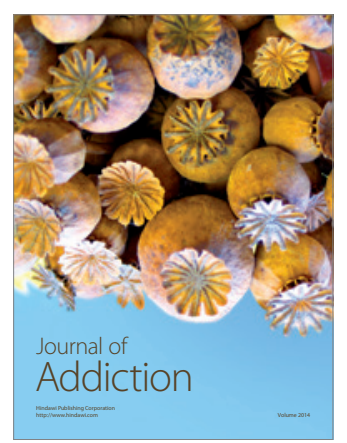

Child Development

Research

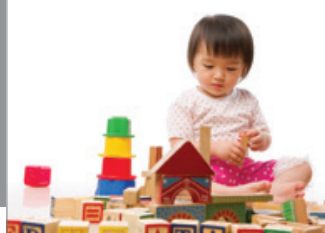

迥
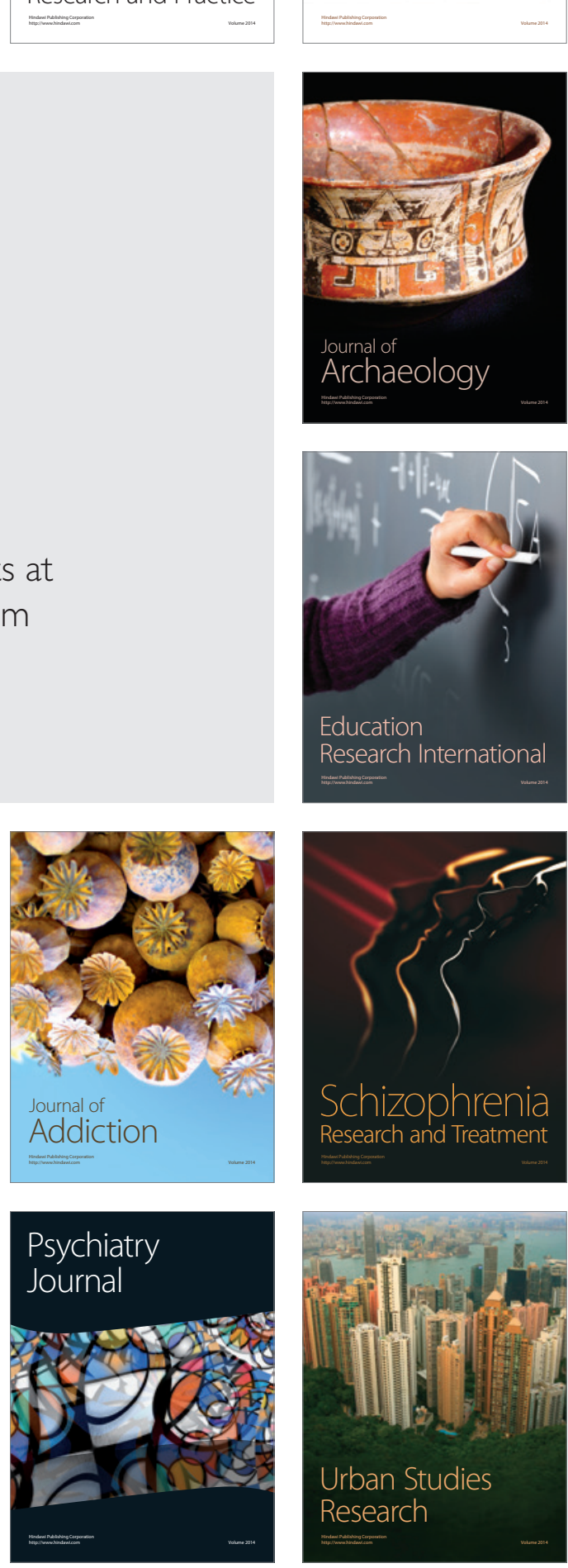